\title{
Survey of Countries in the Geographical Section
}

(Volume 1-5)

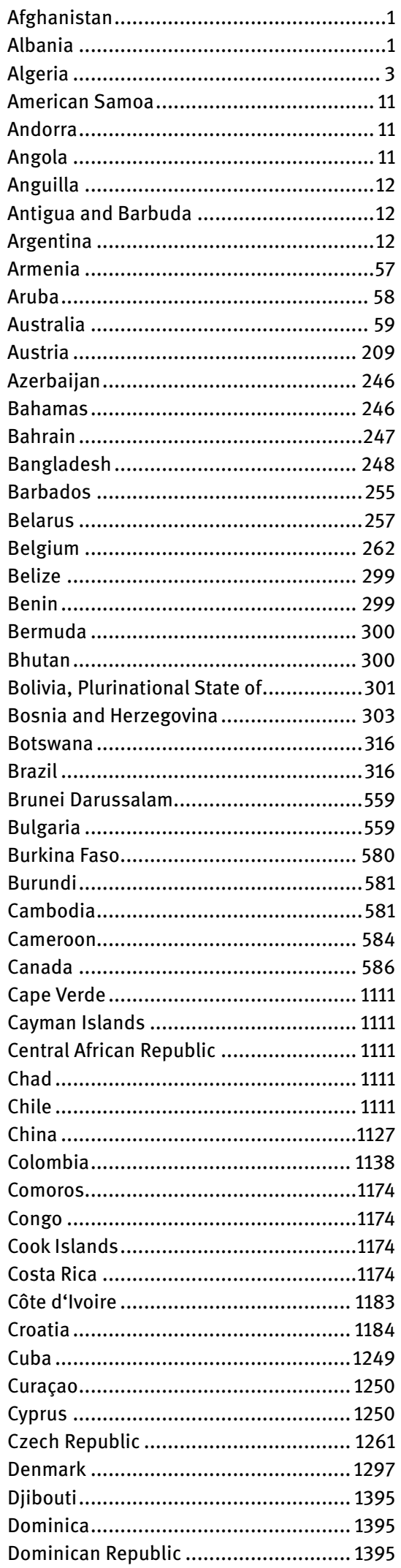

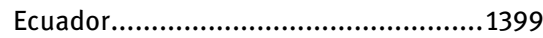

Egypt ................................................... 1401

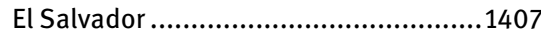

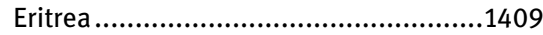

Estonia................................................1409

Ethiopia .............................................. 1413

Falkland Islands (Malvinas)................... 1414

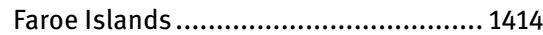

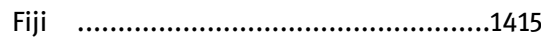

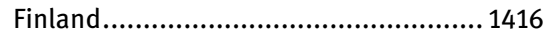

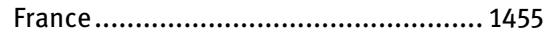

French Guiana ...................................... 1810

French Polynesia................................... 1810

Gabon ................................................1811

Gambia ..............................................1811

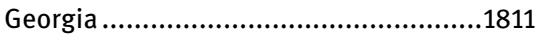

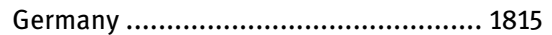

Ghana .............................................. 1977

Gibraltar...........................................1984

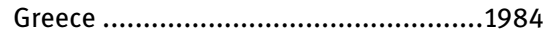

Greenland ..........................................22053

Grenada ...........................................22054

Guadeloupe.....................................2054

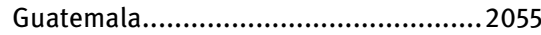

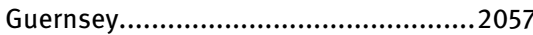

Guinea ..............................................2058

Guyana ............................................22058

Haiti ...................................................2059

Holy See (Vatican City State) .................2059

Honduras ..........................................2059

Hong Kong .........................................2061

Hungary ............................................ 2131

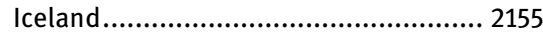

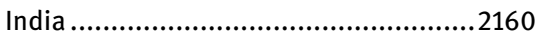

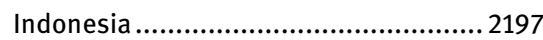

Iran, Islamic Republic of .....................22293

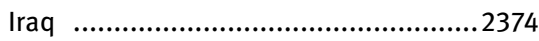

Ireland ..................................................2374

Isle of Man ........................................... 2403

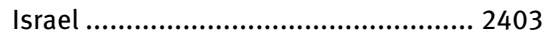

Italy .................................................. 2420

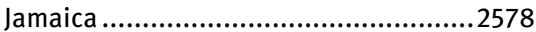

Japan ...................................................2583

Jersey..................................................22691

Jordan ..............................................22691

Kazakhstan ........................................ 2694

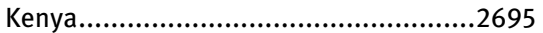

Kiribati.............................................. 2700

Korea, Democratic People‘s

Republic of.

2700

Korea, Republic of ................................. 2701

Kosova .................................................22922

Kuwait................................................ 2926

Kyrgyzstan ....................................... 2926

Lao People‘s Democratic Republic ........22927

Latvia.....

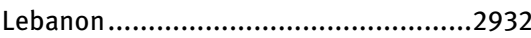

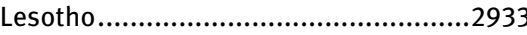

Liberia ..................................................22935

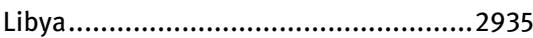

Liechtenstein.................................... 2936

Lithuania........................................... 2936

Luxembourg ....................................... 2951

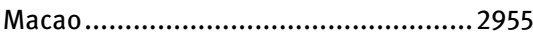

Macedonia, The Former Yugoslav

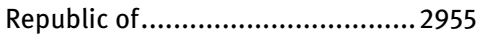

Madagascar........................................ 2962

Malawi ................................................. 2962

Malaysia ........................................... 2963

Maldives .............................................2981

Mali .................................................... 2982

Malta ................................................... 2982

Marshall Islands ................................ 2984

Martinique ....................................... 2984

Mauritania.......................................... 2986

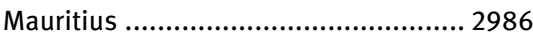

Mayotte............................................... 2987

Mexico …......................................... 2987

Micronesia, Federated States of ............3047

Moldova, Republic of...........................3047

Monaco .................................................3050

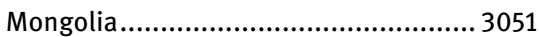

Montenegro........................................3052

Montserrat ......................................... 3060

Morocco ............................................. 3060

Mozambique ........................................ 3071

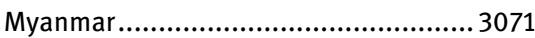

Namibia ............................................... 3071

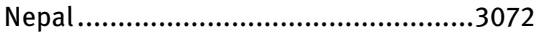

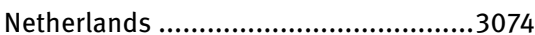

New Caledonia.......................................3232

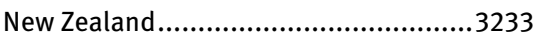

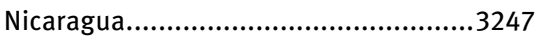

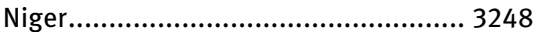

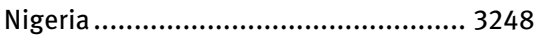

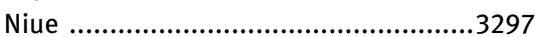

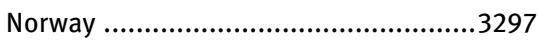

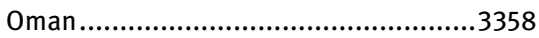

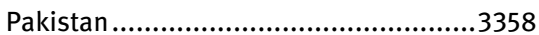

Palau .................................................... 3369

Palestine, State of.............................. 3369

Panama.................................................3370

Papua New Guinea ................................. 3372

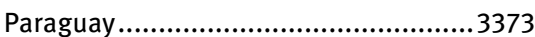

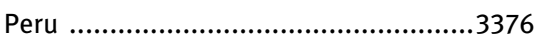

Philippines.........................................3394

Poland ................................................. 3429

Portugal ................................................3656

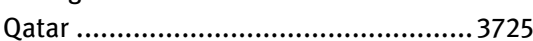

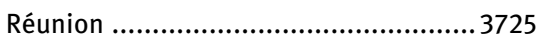

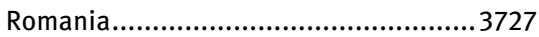

Russian Federation ............................. 3768 
Rwanda.

3876

Saint Helena, Ascension and Tristan

da Cunha

3876

Saint Kitts and Nevis ......................... 3876

Saint Lucia ........................................... 3876

Saint Martin (French part).................... 3876

Saint Pierre and Miquelon .................. 3876

Saint Vincent and The Grenadines .........3877

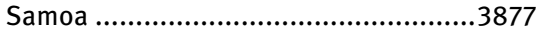

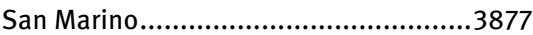

Sao Tome and Principe .........................3877

Saudi Arabia.........................................3877

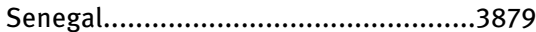

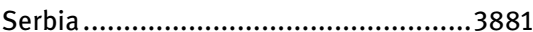

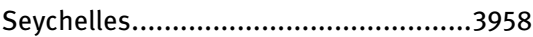

Sierra Leone .......................................3959

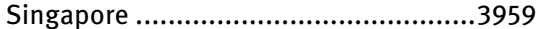

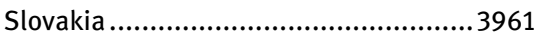

Slovenia...............................................3979
Solomon Islands....................................4001

South Africa ......................................... 4002

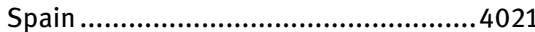

Sri Lanka............................................ 4094

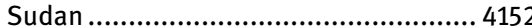

Suriname ............................................. 4152

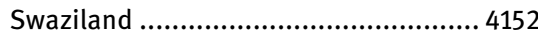

Sweden................................................. 4153

Switzerland ........................................ 4220

Syrian Arab Republic ............................4256

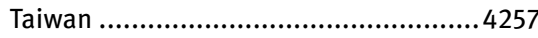

Tajikistan ............................................4297

Tanzania, United Republic of.................4297

Thailand .......................................... 4302

Timor-Leste .........................................4325

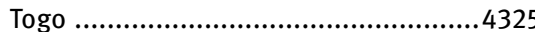

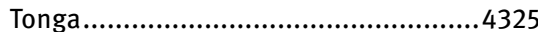

Trinidad and Tobago................................4325

Tunisia ................................................. 4328

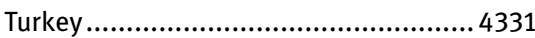

Turks and Caicos Islands .................... 4500

Uganda ............................................... 4500

Ukraine ................................................... 4501

United Arab Emirates ............................. 4521

United Kingdom ...................................... 4521

United States (A-C) .............................. 5051

United States (D-Z) .............................. 5401

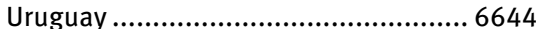

Uzbekistan ......................................... 6647

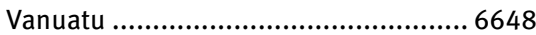

Venezuela, Bolivarian Republic of ....... 6648

Viet Nam ............................................ 6659

Virgin Islands, British......................... 6660

Wallis and Futuna............................... 6660

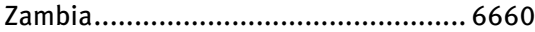

Zimbabwe ...........................................6661 
Geographical Section

Afghanistan - Czech Republic 
